Iranian Journal of Pathology | ISSN: 2345-3656

\title{
Significance of Vascular Endothelial Growth Factor Expression in the Bladder Urothelial Carcinoma and Its Association with Tumor Grade and Invasiveness
}

\author{
Anika Sadaf ${ }^{1}$, Md. Zillur Rahman ${ }^{2}$, Pradip Bhattacharjee', M. Shahab Uddin Ahamad, \\ Sayeeda Nasreen ${ }^{1}$ \\ 1. Department of Pathology, Chittagong Medical College, Chattogram, Bangladesh \\ 2. Department of Pathology, Bangabandhu Sheikh Mujib Medical University, Dhaka, Bangladesh.
}

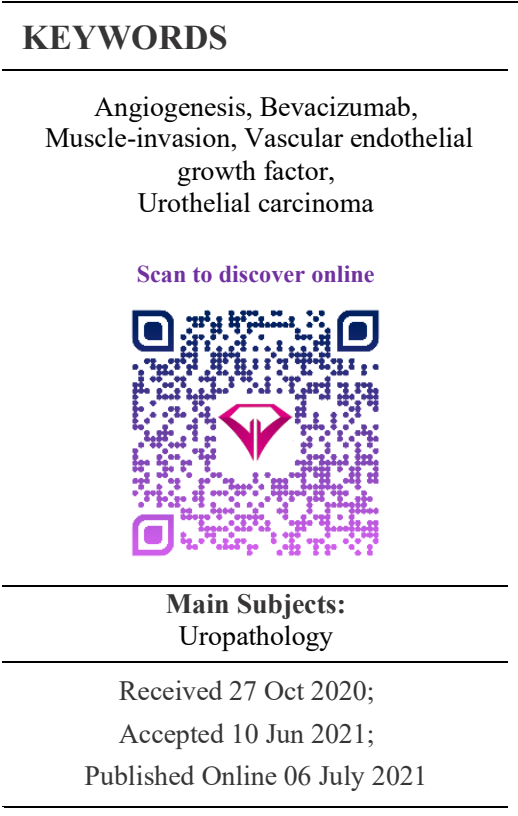

10.30699/IJP.20201.138671.2518

\author{
ABSTRACT
}

Background \& Objective: Vascular Endothelial Growth Factor (VEGF) is one of the newer molecular markers that acts as a central mediator of tumor angiogenesis and is essential for tumor growth, progression, and metastasis. So anti-angiogenic drugs can be used as anticancer therapy. Treatments with anti-VEGF (Bevacizumab) therapy have been proved to improve relapse-free survival in many tumors. Urinary bladder tumor has become emerging cancer globally among elderly individuals. So, the identification and development of novel biomarkers for effective treatment of urinary bladder carcinoma is essential. The present study aimed to investigate the immunohistochemical expression of VEGF in urothelial carcinoma of urinary bladder and to assess its association with tumor grade and muscle invasiveness.

Methods: This cross-sectional study was conducted in the Department of Pathology, Chittagong Medical College, Chattogram from September 2018 to August 2020. Fifty-six formalin-fixed paraffin-embedded tissue blocks of urinary bladder carcinoma were prepared for both histopathological and immunohistochemical examination. Each slide was evaluated by at least two pathologists.

Results: Weak to strong positive expression of VEGF were observed in 52 cases $(92.86 \%)$. The proportion of tumors positive for VEGF expression was higher among patients with high grade and non-muscle invasive bladder carcinoma.

Conclusion: We found that VEGF expression has a significant association with tumor grade and an inverse association with muscle invasion. These findings may be useful for selecting the subset of patients likely to respond to anti-VEGF targeted therapy.

\section{Corresponding Information:}

\section{Dr. Anika Sadaf, Department of Pathology, Chittagong Medical College, Chattogram, Bangladesh}

E-mail: anikasadaf261189@gmail.com

Copyright $\odot$ 2021. This is an open-access article distributed under the terms of the Creative Commons Attribution- 4.0 International License which permits Share, copy and redistribution of the material in any medium or format or adapt, remix, transform, and build upon the material for any purpose, even commercially.

\section{Introduction}

Carcinoma of the urinary bladder is the $10^{\text {th }}$ most common form of cancer worldwide. It is more prevalent in male than in female. The incidence of urinary bladder malignancies in men is 9.6 per 100,000 and the mortality rate is 3.2 per 100,000 worldwide (1). American Cancer Society estimated 60,490 new cases of urinary bladder carcinomas in men and 12,240 deaths among them in the United States in 2017 from population-based data (2). Though the prevalence is higher in developed countries, but incidence is gradually increasing in developing countries like Bangladesh, India etc. due to raising occupational exposure and smoking habit (3).

Bladder cancer generally originates from urothelium and urothelial carcinomas (UC) are the most common type. Approximately $75 \%$ of newly diagnosed patients have non-muscle-invasive bladder cancer (NMIBC) (stage- PTa/ PTis/ PT1) and 25\% have
MIBC or metastatic disease (stage- PT2-PT4) (4). Despite intravesical treatments, 50\%-70\% patients with NMIBC recur within 5 years and up to $30 \%$ progress to MIBC (5). The literature demonstrates the therapeutic and prognostic value of biomarkers involved in the bio-molecular mechanism of urothelial carcinoma and other urinary tract tumors like CXR2 and CXR3 in renal cell carcinoma and prostate-specific antigen (PSA) in prostatic adenocarcinoma (6-11).

Angiogenesis is essential to support the growth of solid tumors. Vascular endothelial growth factor (VEGF) is one of the essential growth factors involved in vasculogenesis and angiogenesis. It is produced by a variety of normal and neoplastic cells and production is regulated by hypoxia. VEGF level varies from high to low in hypoxic to normoxic regions of the tumor. VEGF binds to its cognate receptors, VEGFR1 and VEGFR2 and induce cell proliferation and migration 
(12). It can anticipate the rates of tumor growth and disease outcome to correlate with the abundance of VEGF level. There are evidence to support the existence of VEGF in several cancer types including bladder cancer. In addition, by increasing vascular permeability, VEGF facilitates the entry of tumor cells into circulation and allows them to metastasize to distant sites (13). Many tumor cells and tumor associated vasculature express both VEGF and VEGFRs. The expression of VEGF and its receptors on Kaposi sarcoma, melanoma, ovarian carcinoma, squamous cell carcinoma of the head and neck and breast carcinoma have been reported in last several years (12).

Anti-angiogenic drugs, mainly Bevacizumab, Sorafenib and Sunitinib are already approved for use in many advanced tumors, such as breast, colorectal, liver and kidney cancer; and they are seen to significantly improve the treatment of cancer $(7,8,14)$. Several studies have suggested that inhibition of VEGF transcripts significantly reduced the proliferation rate of the bladder cancer cells (15) and some studies have shown that blockade of VEGF receptor reduced growth and invasion of bladder cancer cells $(16,17)$. Treatment of advanced UC with Bevacizumab (VEGF antibody) and Ramucirumab (VEGFR2 antibody), in combination with chemotherapy showed promising results in phase II clinical trials. A current phase III trial compares Gemcitabine/ Cisplatin (GC) chemotherapy with GC plus Bevacizumab for metastatic or unresectable UC. There are multiple ongoing phase II studies with other agents targeting VEGF receptors, including Sunitinib, Sorafenib and Pazopanib $(18,19)$.

The present study was conducted to observe the expression of VEGF in paraffin embedded tissues taken from histologically confirmed urothelial carcinoma and its association with tumor grade and muscleinvasiveness. So far known, there was no such previous study in Bangladesh. This could be helpful for better understanding regarding the prognostic value of biomarker and the role of anti-VEGF drugs for the effective treatment of urothelial carcinoma.

\section{Material and Methods}

This was a cross sectional observational study conducted in Department of Pathology, Chittagong Medical College, Chattogram, Bangladesh, from September 2018 to August 2020. The authors had prior approval from Institutional Review Board (IRB). Sixty-one consecutive cases of urinary bladder carcinoma were histopathologically examined. Patients other than primary urothelial carcinoma or patients previously treated with chemotherapy or radiotherapy for bladder tumor were excluded. Finally, 56 cases of urothelial carcinomas were prepared for immunohistochemical examination. Data was recorded considering the variables of interest by structured interview and then documented in a pre-designed case record form after taking informed written consent from the patients. Socio-economic profile of the patients was classified according to modified Kuppus-wamy Socioeconomic status scale (20). Patients were eligible for inclusion if they were undergone cystoscopy involved collecting a biopsy or transurethral resection of bladder tumor (TURBT) and all the specimens of each case were submitted for histological examination; and finally diagnosed as primary urothelial carcinoma according to the WHO classification. Tumor grade was evaluated according to 2004 WHO grading system of urothelial tumor. Invasiveness was recorded depending on invasion of the muscularis propria (detrusor muscle) by malignant cells.

\section{Immunohistochemical Evaluation}

The most representative tumor tissue was chosen from each case and 3-5 $\mu \mathrm{m}$ thickness section of formalin fixed paraffin embedded tissues was taken on poly-L-lysine coated slide. After de-paraffinization and rehydration, antigen retrieval was done by microwave at $750^{\circ} \mathrm{C}$ for $15 \mathrm{~min}$. Then the sections were stained with primary antibody against VEGF (monoclonal, dilution 1:50, INVITROGEN, ThermoFisher Scientific, USA) for $30 \mathrm{~min}$. Staining was done using substratechromogen solution: DAB and counterstain was done by $\mathrm{H} \& \mathrm{E}$ staining. VEGF status was scored based on intensity of reactivity and the percentage of reactive cells. Cytoplasm and/or membrane staining was considered as immune-positive. The scoring was done by the 40x objective lens and counting at least 100 cells for immunoreactivity in 10 fields. The results of staining intensity (0-no stain/ colorless, 1-slight staining/ yellowish, 2- moderate staining/ brownyellow, 3-maximal staining/dark brown) and the percentage of positive cells ( 0 - no stain, $1-<25 \%$ positive cells, $2-25-50 \%$ positive cells, 3->50\% positive cells) were added and the final score of immunoexpression for VEGF was recorded as 0: negative $(-), 1-2$ : weak positive $(+), 3-4$ : moderate positive $(++)$, 5-6: strong positive $(+++)$. Renal tubules were used as external control. Omission of the primary antibody was used for negative control.

\section{Statistical Analysis}

SPSS 25 (SPSS Inc., Chicago, Ill., USA) was used for data analysis. Fisher exact test and ANOVA test were performed to find out the possible association of VEGF expression with different variables. P-value $<$ 0.05 was considered statistically significant and confidence interval was set at 95\% level.

\section{Results}

A total of 56 histologically confirmed cases of urothelial carcinomas comprising 48 (85.7\%) males and 8 females were assessed from the TURBT samples. The mean age of the patients was $60.96 \pm 12.93$ years, ranging from 28 to 95 years; male to female ratio was $6: 1$. Most of the patients (47 cases; $83.9 \%$ ) were involved in non-industrial works. Rests were industrial workers; among them, there were 2 leather workers, 2 machinery workers, 2 painters, 2 fertilizer workers and 
1 soap-factory worker. All cases were belonged from low and middle socio-economic class of modified Kuppuswamy Socioeconomic status scale (20). Distribution of the patients according to smoking habits is shown in Table 1.

Regarding past history, six patients $(10.7 \%)$ had history of chronic cystitis, 3 patients (5.4\%) were taking immunosuppressive drugs ( 2 for arthritis and 1 for SLE), 2 patients (3.5\%) had history of instrumenttation for TURP and one had history of both chronic cystitis and immunosuppressive drugs for arthritis.

The site of tumor was assessed through ultrasonography or CT scan. Twenty-two of the tumors $(39.3 \%)$ were found on the right or left lateral wall of the urinary bladder. Rests were on posterior wall (5 cases; $8.9 \%$ ), anterior wall (4 cases; $7.1 \%)$, base ( 3 cases; $5.4 \%$ ), multiple sites (17 cases; $30.4 \%$ ) and as thicken bladder wall ( 5 cases; $8.9 \%$ ).

On microscopic evaluation, high grade (31 cases; $55.4 \%$ ) and MIBC (40 cases; 71.43\%) cases showed predominance. The relationship between different histological grades and muscle invasiveness was found statistically significant $(P<0.05)$ Statistically significant associations $(P<0.05)$ were also evident among VEGF expressions with tumor grade and muscle-invasiveness. Findings obtained from histopathological and immunohistochemical examinations are assembled in Tables 2, $\underline{3}$ and 4 .

Table 1. Distribution of the patients according to smoking habit $(n=56)$

\begin{tabular}{|c|c|}
\hline & Frequency (\%) \\
\hline Smoking habit & \\
\hline Tobacco smoker & $42(75.0)$ \\
\hline Non-tobacco smoker & $14(25.0)$ \\
\hline Duration of smoking (Years) (n=42) & $2(04.76)$ \\
\hline$<\mathbf{1 0}$ & $16(38.10)$ \\
\hline $\mathbf{1 0 - 2 0}$ & $12(28.57)$ \\
\hline $\mathbf{2 1 - 3 0}$ & $12(28.57)$ \\
\hline
\end{tabular}
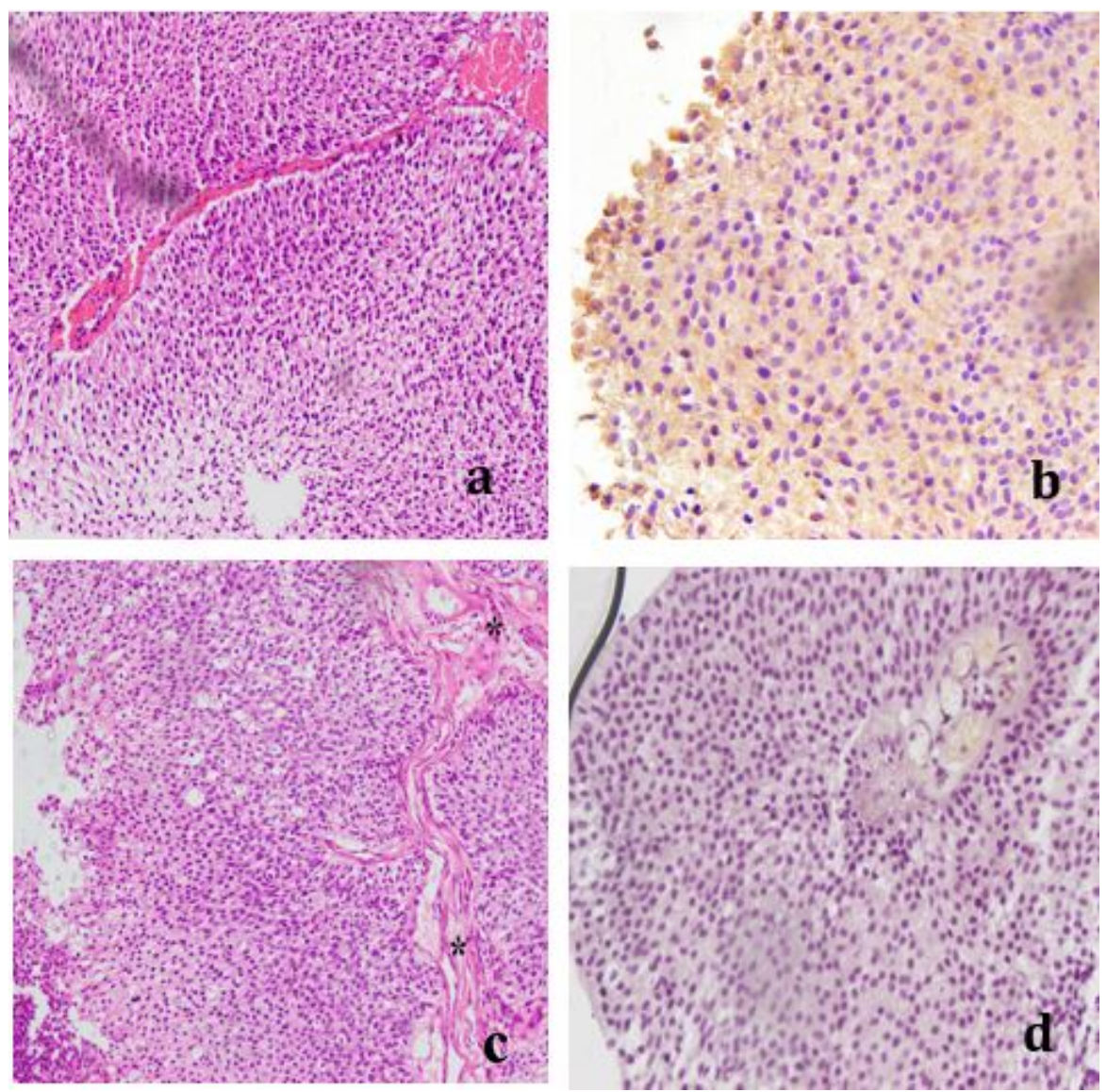

Fig 1. Urothelial carcinoma, Low grade a. NMIBC, H \& E (x20); b. Strong positive (+++) VEGF expression (x40); c. MIBC, H \& E (x20); d. Negative (-) VEGF expression (x40) 
Table 2. Histopathological spectrum of bladder carcinoma $(n=56)$

\begin{tabular}{|c|c|}
\hline & Frequency (\%) \\
\hline Histopathological grade & $31(55.4)$ \\
\hline High & $25(44.6)$ \\
\hline Low & $40(71.43)$ \\
\hline Muscle invasiveness & $9(16.07)$ \\
\hline MIBC & $7(12.50)$ \\
\hline NMIBC & \\
\hline Muscle could not be identified & \\
\hline
\end{tabular}

MIBC: Muscle invasive bladder carcinoma, NMIBC: Non-muscle invasive bladder carcinoma

Table 3. Relationship between histological grade and muscle invasiveness $(n=49)$

\begin{tabular}{|c|c|c|c|c|}
\hline \multirow{2}{*}{ Muscle invasiveness } & \multicolumn{2}{|c|}{ Histopathological grade } & \multirow{2}{*}{ Total } & \multirow{2}{*}{ P-value* } \\
\cline { 2 - 4 } & Low & High & 09 & \multirow{2}{*}{$\mathbf{0 . 0 0 0 2}^{\text {s }}$} \\
\hline NMIBC & $09(40.9)$ & $00(0.0)$ & 40 & $49^{\Psi}$ \\
\hline MIBC & $13(59.1)$ & $27(100.0)$ & $27(100.0)$ & \\
\hline
\end{tabular}

*Fisher exact test was done to measure the level of significance.

${ }^{\Psi}$ Seven cases were excluded as muscle could not be identified

Table 4. Expression of VEGF in among grades and muscle-invasiveness of bladder carcinoma

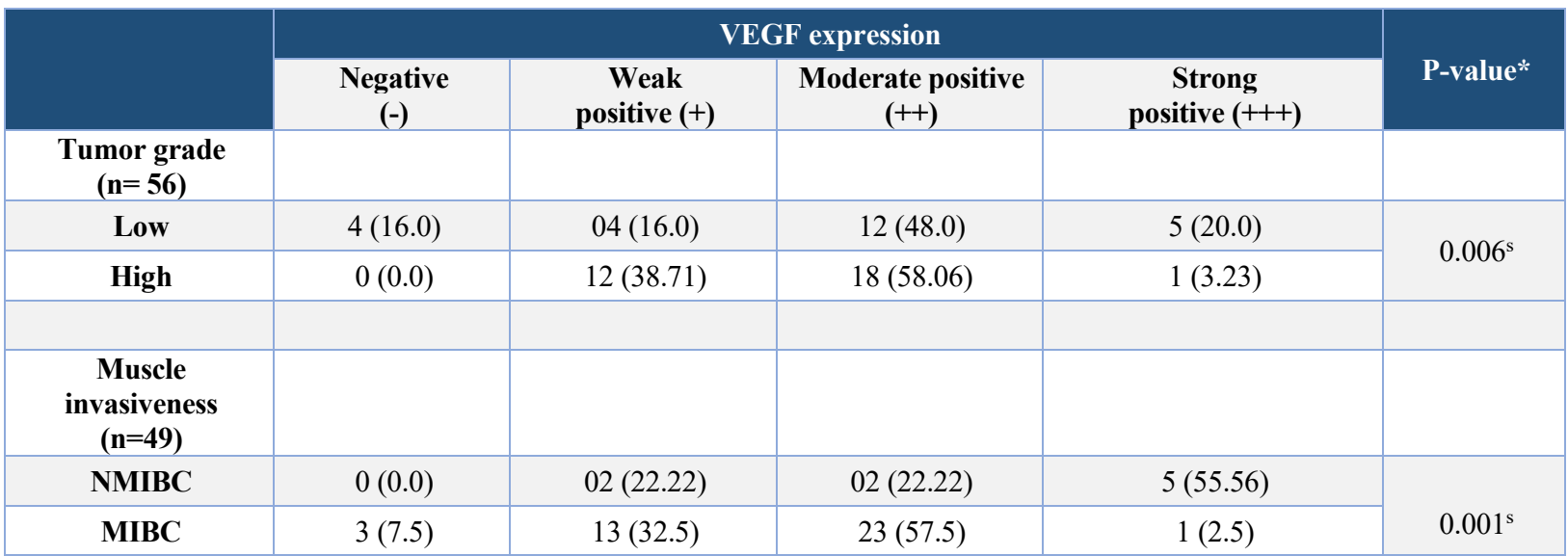

*Fisher exact test was done to measure the level of significance. Figure within parenthesis indicates in percentage; $\mathrm{s}=$ statistically significant

Table 5. Comparison of VEGF expression in UBC among different studies:

\begin{tabular}{|c|c|c|c|c|}
\hline \multirow{2}{*}{ Author } & \multirow{2}{*}{ Country } & \multirow{2}{*}{$\begin{array}{c}\text { Total number of } \\
\text { cases }\end{array}$} & \multicolumn{2}{|c|}{ VEGF expression } \\
\hline & & & Positive & Negative \\
\hline Yang et al. (27) 2004 & Taiwan & 161 & $54.70 \%$ & $45.30 \%$ \\
\hline Xia et al. (12) 2006 & California, USA & 72 & $97.20 \%$ & $2.80 \%$ \\
\hline Rahmani et al. (28) 2012 & New Delhi, India & 125 & $43.20 \%$ & $56.80 \%$ \\
\hline Kopparapu et al. (29) 2013 & Philadelphia, USA & 212 & $79.20 \%$ & $20.80 \%$ \\
\hline Adelmann et al. (30) 2018 & Romania & 50 & $13.33 \%$ & $86.67 \%$ \\
\hline Present study 2020 & Bangladesh & 56 & $92.86 \%$ & $07.14 \%$ \\
\hline
\end{tabular}



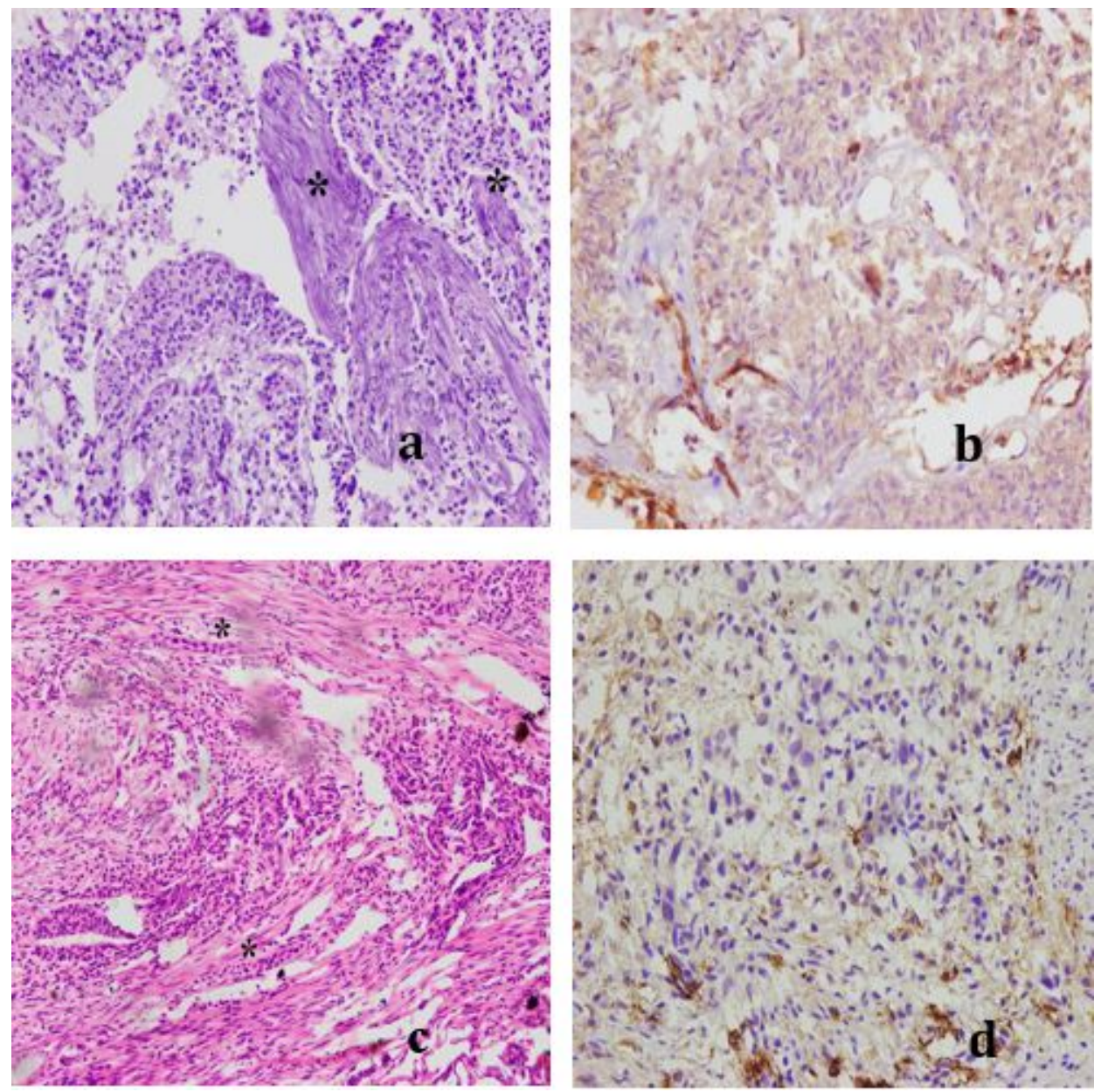

Fig 2. Urothelial carcinoma, High grade. a. MIBC, H \& E (x20); b. Moderate positive (++) VEGF expression (x40); c. MIBC, H \& E (x20); d. Weak positive (+) VEGF expression (x40) Asterisk (*) marks in MIBC cases indicate invasion of muscularis propria by malignant cells

\section{Discussion}

Present study showed male predominance $(48$ cases; $85.74 \%$ ) with $6: 1$ ratio. Gupta et al. in 2009 , found a male to female ratio of $8.6: 1$ (21). The higher incidence of urinary bladder carcinoma in male may be due to the personal habit such as smoking and more exposure to carcinogenic agents due to their occupation. Rushton et al. in 2010 described that $5.6 \%$ urinary bladder malignancies were attributable to occupational exposure in their study (22).

We observed that, the incidence of high-grade urothelial carcinoma (HGUC) was higher in this study. Haque et al. (2018) recorded $72.0 \%$ patients with HGUC and remaining with low grade urothelial carcinoma (LGUC) (23). The study conducted by Chinnasamy et al. (2016) in India observed 63.4\% cases of high-grade carcinoma and by the one performed by Chou et al. (2013) in Taiwan found $56.8 \%$ cases of high-grade carcinoma $(24,25)$. All these studies were in accordance with present study.

Current study found 40 cases $(71.43 \%$ ) with MIBC and 9 cases $(16.07 \%)$ with NMIBC; while in rest of the cases ( 7 cases; $12.50 \%$ ) muscularis propria could not

be identified. Chou et al. in 2013 also found high incidence of MIBC; revealing $40.5 \%$ NMIBC and $59.5 \%$ MIBC (25). On the other hand, Chinnasamy et $a l$. in 2016 recorded $86.5 \%$ cases of NMIBC (stagePT1) and only $13.5 \%$ cases of MIBC (stage- PT2) (24). According to Thapa et al. in 2017 muscle invasion was observed in $24.45 \%$ cases of high-grade urothelial carcinomas; however, none of the cases of low grade papillary urothelial carcinoma showed muscle invasion (26). In current study, among twenty-two low grade carcinoma cases $(\mathrm{n}=49), 9$ cases $(40.9 \%)$ were NMIBC and 13 cases (59.1\%) were MIBC. On the other hand, among 27 cases of high-grade carcinomas $(n=49)$, all were MIBC. These differences between different tumor grades and muscle invasiveness were found statistically significant $(P<0.05)$. Incidence of MIBC was high in our study. Poor socio-economic condition, lack of awareness, inadequate facilities of routine check-up for elderly people as well as social and religious restrictions in our country predispose to delayed healthcare seeking and may be the probable causes of increased rate of MIBC. 
Our primary aim was to observe the immunohistochemical expression of VEGF in urinary bladder carcinoma. Table 5 shows the percentage of VEGF expression in urinary bladder carcinomas observed in different studies conducted in different countries.

All the cases of high-grade carcinomas (31 cases) showed weak $(+)$ to strong $(+++)$ positive VEGF expression and none showed negative (-) expression. On the other hand, proportion of negative (-) VEGF expression was significantly higher among patients with low grade carcinoma. This indicated that with the progression in tumor grade, the rate of VEGF expression significantly increased $(P<0.05)$; which was similar to Yang et al. (2004) and Rahmani et al. (2012) $(27,28)$.

This study also evaluated the association between VEGF expression and muscle-invasiveness of urinary bladder carcinomas and revealed that all NMIBC cases were positive for VEGF expression and 55.56\% (5 cases) showed strong positive $(+++)$ expression. Whereas only $2.5 \%$ ( 1 case) of MIBC cases showed strong positive $(+++)$ VEGF expression and $7.5 \%$ (3 cases) were negative (-) for VEGF expression. Statistically significant $(P<0.05)$ differences were evident between VEGF expressions and muscleinvasiveness. Kopparapu et al. (2013) showed that VEGF expression was significantly higher in NMIBC compared to MIBC. The rate of tumor growth is higher at the early stage of the disease, which may be anticipated by VEGF. Thus, NMIBC may be correlated with the abundance of VEGF (29). Yang et al. in 2004 observed that the rate of positive expression of VEGF was significantly high in MIBC and furthermore, was associated with poor prognosis as seen in a 5-year follow up. So, they recommended VEGF as a useful prognostic marker for TCC (27). Özveren and Türkeri in 2019 observed that, the proportion of tumors positive for VEGF expression was higher in patients with NMIBC (45\% vs $31 \%$ ) and in high grade tumors
(44\% vs $37.5 \%$ ). But statistically significant difference was not found in VEGF mRNA positivity with muscleinvasiveness by RT-PCR analysis of fresh tumor tissues (31).

\section{Limitation}

Information regarding extra-vesicular extension, lymph node and distal metastasis could not be included due to lack of logistic support, which could give more conclusive decision regarding prognostic significance of VEGF.

\section{Conclusion}

The data obtained in the present study led us to conclude that the proportion of tumors positive for VEGF expression was higher among patients with high grade and non-muscle invasive bladder carcinoma. The expression profile of VEGF may be useful for selecting high risk patients and predicting the subset of patients likely to response to anti- VEGF targeted therapy.

\section{Acknowledgements}

Special thanks to Dr. Mohammad Ismail Hossain, Lecturer, Department of Pathology, Chittagong Medical College, Chattogram for his enormous support and advice. We are grateful to all the doctors of Department of Urology, Chittagong Medical College Hospital, Chattogram; without their kind support this study would not have been possible. We specially acknowledge all the patients from whom specimen were collected and their attendants, for their active participation in this study.

\section{Conflict of Interest}

The authors declared no conflict of interest.

\section{References}

1. Bray F, Ferlay J, Soerjomataram I, Rebecca L, Lindsey A, Jemal A. Global cancer statistics 2018: GLOBOCAN estimates of incidence and mortality worldwide for 36 cancers in 185 countries. CA Cancer J Clin.2018; 68(6):394-424 [DOI:10.3322/caac.21492] [PMID]

2. Siegel RL, Miller KD, Jemal A. Cancer statistics, 2017. CA Cancer J Clin 2017; 67(1):7-30 [DOI:10.3322/caac.21387] [PMID]

3. Cumberbatch MGK, Cox A, Teare D, Catto JWF. Contemporary occupational carcinogen exposure and bladder cancer. JAMA Oncol. 2015; 1: 1282-90. [DOI:10.1001/jamaoncol.2015.3209] [PMID]
4. Sanli O, Dobruch J, Knowles MA, Burger M, Alemozaffar M, Nielsen ME et al. Bladder cancer. Nat Rev Dis Primers. 2017; 3(17022): 1-19. [DOI:10.1038/nrdp.2017.22] [PMID]

5. Jacobs BL, Lee CT, Montie JE. Bladder cancer in 2010: How far have we come? Ca Cancer J Clin 2010; 60:244-272. [DOI:10.3322/caac.20077] [PMID]

6. Zhao J, Xu W, Zhang Z, Song R, Zeng S, Sun Y, Xu C. Prognostic role of HER2 expression in bladder cancer: a systematic review and meta-analysis. Int Urol Nephrol. 2015; 47:87-94. [DOI:10.1007/s11255-0140866-z] [PMID] 
7. Elfiky AA, Rosenberg JE. Targeting angiogenesis in bladder cancer. Current Oncology Report 2009; 11:244-249. [DOI:10.1007/s11912-009-0034-2] [PMID]

8. Abdalla AM, XiaoL, Ullah MW, Yu M, Ouyang C, Yang G. Current challenges of cancer Anti-angiogenic therapy and the promise of Nano therapeutics. Theranostics 2018; 8(2): $\quad 533-\quad 48$ [DOI:10.7150/thno.21674] [PMID] [PMCID]

9. Kim WJ, Bae SC. Molecular biomarkers in urothelial bladder cancer. Cancer Sci. 2008; 99(4):646-52. [DOI:10.1111/j.1349-7006.2008.00735.x] [PMID]

10. Rezakhaniha B, Dormanesh B, Pirasteh H, Yahaghi E, Masoumi B, Ziari K, Rahmani O. Immunohistochemical distinction of metastases of renal cell carcinoma with molecular analysis of overexpression of the chemokines CXCR2 and CXCR3 as independent positive prognostic factors for the tumorigenesis. Int Uni Biochem and Mol Bio. 2016; 68(8):629-33. [DOI:10.1002/iub.1520] [PMID]

11. Rezakhaniha B, Pour, Arian N, Siroosbakhat S. Effect of cystoscopy on Prostate-Specific Antigen, new words about old subject. Iran J Cancer Prev. 2010; 3(4):19398.

12. Xia G, Kumar SR, Hawes D, Cai J, Hassanieh L, Groshen $\mathrm{S}$ et al. Expression and significance of vascular endothelial growth factor receptor 2 in bladder cancer. J Urol. 2006; 175:1245-52. [DOI:10.1016/S0022-5347(05)00736-6]

13. Slaton JW, Millikan R, Inoue $\mathrm{K}$, Karashima $\mathrm{T}$, Czerniak B, Shen Yet al. Correlation of metastasis related gene expression and relapse-free survival in patients with locally advanced bladder cancer treated with cystectomy and chemotherapy. J Urol. 2004; 171: 570-74. [DOI:10.1097/01.ju.0000108845.91485.20] [PMID]

14. Roviello G, Bachelot T, Hudis CA, Curigliano G, Reynolds AR, Petrioli R et al.The role of bevacizumab in solid tumours: A literature based meta-analysis of randomised trials. Eur J Cancer. 2017; 75:245-258. [DOI:10.1016/j.ejca.2017.01.026] [PMID]

15. Kunze D, Wuttig D, Kausch I, Blietz C, Blumhoff L, Burmeister $\mathrm{Y}$ et al. Antisense-mediated inhibition of survivin, hTERT and VEGF in bladder cancer cells in vitro and in vivo. Int $\mathrm{J}$ Oncol. 2008; 32: 1049- 56. [DOI:10.3892/ijo.32.5.1049] [PMID]

16. Nakanishi R, Oka N, Nakatsuji H, Koizumi T, Sakaki $\mathrm{M}$, Takahashi $\mathrm{M}$ et al. Effect of vascular endothelial growth factor and its receptor inhibitor on proliferation and invasion in bladder cancer. Uro Int. 2009; 83:98106. [DOI:10.1159/000224877] [PMID]

17. Videira PA, Piteira AR, Cabral MG, Martins C, Correia $\mathrm{M}$, Severino $\mathrm{P}$ et al. Effects of bevacizumab on autocrine VEGF stimulation in bladder cancer cell lines. UrolInt. 2011;86: 95-101. [DOI:10.1159/000321905] [PMID]
18. Fus ŁP, Górnicka B. Role of angiogenesis in urothelial bladder carcinoma. Cent European J Urol. 2016; 69: 258-263.

19. Schulz GB,Karl A. The value of anti-angiogenicsin bladder cancer therapy, in Marmé, D. ed.Tumor Angiogenesis: A Key Target for Cancer Therapy. 2017; Cham, Springer International Publishing: 1-13. [DOI:10.1007/978-3-319-31215-6 36-1]

20. Ahmed B, Shiraji KH, Chowdhury MHK, Uddin MG, Islam SN, Hossain S. Socio-economic status of the patients with acute coronary syndrome: Data from a district-level general hospital of Bangladesh. Cardiovasc J. 2017; 10(1): 17-20. [DOI:10.3329/cardio.v10i1.34357]

21. Gupta P, Jain M, Kapoor R, Muruganandham K, Srivastava A, Mandhani A. Impact of age and gender on the clinicopathological characteristics of bladder cancer. Indian J Urol. 2009;25(2): 207-10. [DOI:10.4103/0970-1591.52916] [PMID] [PMCID]

22. Rushton L, Bagga S, Bevan R, Brown TP, Cherrie JW, Holmes Pet al. Occupation and cancer in Britain. B J Cancer. 2010; 102: 1428-37. [DOI:10.1038/sj.bjc.6605637] [PMID] [PMCID]

23. Haque S, Dewan RK, Saleh S, JennahSA, Jahan F, Akter $\mathrm{F}$ et al. Histomorphological study of urinary bladder tumor and status of HER2/Neu and Ki67 expression in urothelial carcinoma. J Histopathol Cytopathol. 2018 July;2(2): 99-108.

24. Chinnasamy R, Krishnamoorthy S, Josheph L, Kumaresan N, Ramanan V. Clinico-pathological study of bladder cancer in a tertiary care center of South India and impact of age, gender and tobacco in causing bladder cancer: A single center experience. Int J Sci Stu. 2016; 3(10): 72-77.

25. Chou YH, Chang WC, Wu W, Li CC, Yeh HC, Hou $\mathrm{MF}$ et al. The association between gender and outcome of patients with upper tract urothelial cancer. Kaohsiung J Med Sci. 2013; 29(1):37-42. [DOI:10.1016/j.kjms.2012.08.006] [PMID]

26. Thapa R, Lakhey M, Bhatta AD. Spectrum of histomorphological diagnosis in cystoscopic bladder biopsies. J Pathol Nepal. 2017; 7(1):1062-65. [DOI:10.3126/jpn.v7i1.16913]

27. Yang CC, Chub KC, Yeh WM. The expression of vascular endothelial growth factor in transitional cell carcinoma of urinary bladder is correlated with cancer progression. Urol Oncol. 2004;22:1-6. [DOI:10.1016/S1078-1439(03)00015-2]

28. Rahmani A, Alzohairy M, Khadri H, Mandal AK, Rizvi MA. Expressional evaluation of Vascular EndothelialGrowth Factor (VEGF) protein in urinary bladder carcinoma patients exposed to cigarette smoke. Int J Clin Exp Pathol. 2012; 5(3):195-202. 
29. Kopparapu PK, Boorjian SA, Robinson BD, Downes M,Gudas LJ, Nigel PM,Persson JL. Expression of VEGF and its receptors VEGFR1/ VEGFR2 is associated with invasiveness of bladder cancer. Anticancer Research. 2013; 33, 2381-90.

30. Adelmann T, Ioiart I, Ceausu R, Sarb S, Suciu C. Immunohistochemical expression of Vascular Endothelial Growth Factor does not correlate with microvessel density in invasive bladder carcinoma. Euro J Innov Integrat Trans Med. 2018; 2(1): 12-17.
31. Özveren B, Türkeri L. Vascular Endothelial Growth Factor and Thrombospondin-1 mRNA expression in bladder tumors: Correlation with histopathology and prognosis. Bull Urooncol. 2019;18: 18-23. [DOI:10.4274/uob.galenos.2018.1179]

\section{How to Cite This Article}

Sadaf, A., Rahman, Z., Bhattacharjee, P., Uddin Ahamad, M, Sh., Nasreen, S Significance of Vascular Endothelial Growth Factor Expression in the Bladder Urothelial Carcinoma and Its Association with Tumor Grade and Invasiveness Iran J Pathol, 2021; 16(4): 362-369. doi: 10.30699/IJP.2021.138671.2518 